\title{
Neuropsychological Follow-up After Neonatal ECMO
}

Raisa M. Schiller MSc ${ }^{1}$, Marlous J. Madderom $\mathrm{PhD}^{1}$, Jolanda J.C.M. Reuser MSc ${ }^{2}$, Katerina Steiner $\mathrm{MD}^{3}$, Saskia J. Gischler MD $\mathrm{PhD}^{1}$, Dick Tibboel MD PhD ${ }^{1}$, Arno van Heijst MD PhD" Hanneke IJsselstijn MD $\mathrm{PhD}^{1}$

Affiliations: ${ }^{1}$ Intensive Care and Department of Pediatric Surgery, Erasmus MC-Sophia Children's Hospital, Rotterdam, the Netherlands; ${ }^{2}$ Department of Medical Psychology, Radboud University Medical Center, Nijmegen, the Netherlands; and ${ }^{3}$ Department of Neonatology, Radboud University Medical Center, Nijmegen, the Netherlands

Corresponding author: H. IJsselstijn Intensive Care and Department of Pediatric Surgery Erasmus Medical Center-Sophia Children's Hospital Room Sk-1280 dr. Molewaterplein 60, 3015 GJ Rotterdam, the Netherlands Email: h.ijsselstijn@erasmusmc.nl Telephone: +31.10.7036203

Short title: Neuropsychological Follow-up After Neonatal ECMO

Financial Disclosure: The authors have no financial relationships relevant to this article to disclose.

Funding source: Raisa Schiller was funded by the Sophia Stichting Wetenschappelijk Onderzoek (SSWO): S14-213.

Conflict of Interest: The authors have no conflicts of interest relevant to this article to disclose.

Abbreviations: ECMO, extracorporeal membrane oxygenation; MDI, mental developmental index; BOS 2-30, Bayley Developmental Scales; BSID-II-NL, the Bailey Scales of Infant Development - Second Edition - Dutch version; RAKIT, The Revised Amsterdam Intelligence Test; WISC-III-NL, Wechsler Intelligence Scale for children; VA, venoarterial; VV, venovenous; CLD, chronic lung disease; MAS, meconium aspiration syndrome; $\mathrm{CDH}$, congenital diaphragmatic hernia.

\section{What's Known on This Subject}

Neonatal ECMO survivors are at risk for long-term neuropsychological impairments and school problems, while cross-sectional studies have shown intelligence to be average. Despite increased awareness of these problems, neonatal ECMO follow-up protocols are not equipped to detect these impairments.

\section{What This Study Adds}

Longitudinal evaluation of intelligence in neonatal ECMO survivors shows stable and average IQ at 2-5-8 years. School problems following neonatal ECMO are related to worse selective attention, irrespective of IQ. Congenital diaphragmatic hernia patients are at highest risk. 


\section{Contributors' Statements:}

Ms. Schiller and dr. Madderom carried out the analyses, drafted the initial manuscript, reviewed and revised the manuscript, and approved the final manuscript as submitted.

Dr. Gischler, dr. van Heijst, prof. Tibboel and dr. IJsselstijn conceptualized and designed the study, reviewed and revised the manuscript, and approved the final manuscript as submitted.

Ms. Reuser and dr. Steiner contributed to acquisition of the data, reviewed and revised the manuscript, and approved the final manuscript as submitted. 


\begin{abstract}
Objective To assess the longitudinal development of intelligence and its relation to school performance in a nationwide cohort of neonatal ECMO survivors as well as evaluate predictors of outcome at eight years.

Methods Repeated measurements of intelligence in neonatal ECMO survivors were collected at two, five and eight years $(\mathrm{n}=178)$ with validated, standardized instruments. Selective attention $(\mathrm{n}=148)$ and type of education were evaluated in the eight-year-olds.

Results Intelligence was found to remain stable and average across development (mean IQ(SD) at $2=102(18)$; at $5=100(17)$; at $8=99(17)), p=.15$. Children attending regular education without the need for help ( $n=101$, mean $\mathrm{z}$-score(SD)=-1.50(1.93)) performed significantly better on the selective attention task compared to those children in need of extra help ( $\mathrm{n}=65$, mean $\mathrm{z}-$ score $(\mathrm{SD})=-2.54(3.18)$ ) or those attending special education $(\mathrm{n}=13$, mean $\mathrm{z}$-score(SD)=4.14(3.63)), $p=.03$. However, only children attending special education had below average intelligence (mean IQ(SD)=76(15)), compared to average intelligence for those attending regular education, both with (mean IQ(SD)=95(15)) and without help (mean IQ(SD)=105(16)). Children with congenital diaphragmatic hernia scored significantly lower on both IQ (CDH mean $\mathrm{IQ}(\mathrm{SD})=93(20)$; MAS mean $\mathrm{IQ}(\mathrm{SD})=100(15)$; other diagnoses mean $\mathrm{IQ}(\mathrm{SD})=100(19), p=.04)$ and selective attention (CDH, mean z-score(SD)=-3.48(3.46)); MAS mean z-score(SD)=$1.60(2.13)$; other diagnoses mean $\mathrm{z}$-score(SD)=-1.65(2.39), $p=.002$ ) compared to other diagnoses.

Conclusion Intelligence testing alone does not identify those at risk for academic problems for the majority of neonatal ECMO survivors. We propose internationally standardized follow-up protocols that focus on long-term problem-oriented neuropsychological assessment.
\end{abstract}




\section{Introduction}

Extracorporeal membrane oxygenation (ECMO) has been used in over 28,000 neonates with severe respiratory failure who are unresponsive to conventional medical management ${ }^{1}$. Survival rates have remained stable over the years with 5-10\% surviving with severe neurological complications $^{1}$. The remaining $90 \%$ of survivors are at risk for subtler long-term neurodevelopmental problems ${ }^{2-4}$. Despite increasing awareness of these problems, the current standardized (international) follow-up protocols are inadequate for the detection of neuropsychological deficits in neonatal ECMO survivors ${ }^{1,5}$. As the ELSO recommendations have not been reviewed since 1997, an evidence-based update is mandatory ${ }^{6}$.

In many follow-up programs, intelligence remains the primary outcome measure ${ }^{5,6}$. Previous studies have shown intelligence to be comparable to that of healthy children at various stages of development ${ }^{2,3,7-9}$. IQ testing can give valuable insight into the overall cognitive functioning of an individual, but is not suited to detect subtle neuropsychological impairments ${ }^{10}$. Extensive neuropsychological testing in neonatal ECMO survivors has demonstrated deficits especially in the attention and (working) memory domains in 8- and 17-year-olds ${ }^{2,4}$ with an increased need for extra help in school ${ }^{2,4,8}$. Since IQ is generally within the average range, the school problems are likely due specific neuropsychological impairments. However, this remains largely speculative and IQ has not been studied longitudinally.

In this study, we aimed to investigate the relationship between school problems and cognitive outcome in neonatal ECMO survivors. To do so, we first assessed the longitudinal development of intelligence at two, five and eight years of age in a nationwide cohort of neonatal ECMO survivors. We then evaluated type of education attendance in relation to intelligence and to selective attention at eight years of age. Finally, we studied whether school performance and cognitive outcome at eight years of age were influenced by clinical characteristics. We hypothesized that intelligence is normal across the three ages and unrelated to the school problems observed in neonatal ECMO survivors. Based on this, we propose standardized, problem-oriented follow-up aimed at specific neuropsychological domains that can be internationally implemented. 


\section{Methods}

Population

Patients born between January 1996 and December 2006 treated with ECMO within the first 28 days of life and participating in the structured prospective post-ECMO follow-up program were eligible for the current study $(n=278)$. Children were either part of the follow-up program that was initiated in 2001 at the Erasmus MC-Sophia Children's Hospital in Rotterdam ( $\mathrm{n}=143)$ or at the Radboud University Medical Centre in Nijmegen initiated in 1998 ( $\mathrm{n}=135)$. ECMO support was given according to the criteria described by Stolar et $\mathrm{al}^{11}$ which did not change over time. Entry and exclusion criteria for follow-up were previously described ${ }^{2,7}$. The post-ECMO follow-up program is the standard of care in the Netherlands ${ }^{2,7,12}$, therefore Institutional Review Board approval was waived. Only those children of whom at least the mental developmental index at two and IQ at eight years of age were evaluated were included (Rotterdam, $n=96$, Nijmegen, $n=82$ ) (Figure 1). Demographic and medical characteristics of the patients are reported in Table 1.

\section{Neuropsychological assessment}

\section{Intelligence}

Intelligence was measured at two, five and eight years of age. For two-year-olds, the Bailey Developmental Scales (BOS 2-30) $(n=100)$ or, from December 2003, the Bailey Scales of Infant Development - Second Edition - Dutch version (BSID-II-NL) $(n=78)$ were used to assess mental outcome. These standardized instruments both assess verbal and non-verbal development of 2-to-30 month-old children and are substantially related to each other ${ }^{13}$.

The Revised Amsterdam Intelligence Test (RAKIT) short-form was used at five years ${ }^{14}$. For the eight-year-olds, the RAKIT $(n=102)$ or the Wechsler Intelligence Scale for children (WISC-III-NL; $n=76$ ) was used ${ }^{15}$. Both tests assess verbal and non-verbal intelligence, have been shown to have good reliability and validity ${ }^{14,15}$, and have been used interchangeably by our group before $^{16}$.

For all four tests, a normalized population mean of 100 with a standard deviation of 15 is used $^{13-15}$. The outcome on all four tests is referred to as intelligence or IQ.

\section{$\underline{\text { Selective attention }}$}

Selective attention was measured in the eight-year-old children $(n=148)$ with the Dot Cancellation paper-and-pencil test. The main outcome measure was working-speed, which was converted into z-scores (individual score minus the population score divided by the population standard deviation). Good validity, sensitivity, reliability and Dutch normative data have been reported $^{17}$.

\section{Procedures and study design}

Children underwent neuropsychological evaluation by a psychologist at two, five and eight years of age. Parents filled in questionnaires on ethnicity (Dutch/ $\geq 1$ non-native Dutch parent) and maternal educational level (MEL; high/moderate/low). MEL refers to the highest type of education completed by the mother ${ }^{18}$. Various medical characteristics were recorded prospectively: birth weight, gestational age, diagnosis, age at the start of ECMO, ECMO duration/type (venoarterial (VA)/venovenous(VV)/VV conversion to VA), duration of mechanical ventilation, extra oxygen supply post extubation, chronic lung disease (CLD; 
yes $/$ no) ${ }^{19}$ and abnormal cranial ultrasound (CUS; yes (i.e. parenchymal or intracranial bleedings)/no).

\section{Data analysis}

Clinical characteristics of participants and non-participants of the follow-up program were compared using independent-samples T-tests for the normally distributed data and MannWhitney U tests for the non-normally distributed data.

The developmental trajectory of intelligence was evaluated using repeated-measures ANOVA. Normality tests were performed. Mauchly's test was used to assess and correct for sphericity.

Type of education attendance at eight years of age and its relations to intelligence at two, five and eight and selective attention at eight were analyzed using Kruskal-Wallis $\mathrm{H}$ tests. For post-hoc analyses, the three education categories were transformed into two dummy variables: 1 ) regular education with help versus regular education and special education and 2) special education versus regular education with and without help. Independent samples T-tests were then conducted to evaluate which groups differed.

Next, the effect of diagnosis (meconium aspiration syndrome (MAS), congenital diaphragmatic hernia (CDH), other diagnoses) on intelligence at all ages, and selective attention at eight years were evaluated using Kruskall-Wallis $\mathrm{H}$ tests, as previous research has shown $\mathrm{CDH}$ patients to perform worse compared to children with other diagnoses ${ }^{2}$.

Finally, associations between IQ at two years, IQ at five years and outcome at eight years of age were evaluated using multivariate linear regression analyses, adjusted for MEL and parents' ethnicity $^{20-22}$. Parents' ethnicity was used because a child's verbal skills, and thus neurodevelopmental outcome, may be affected by a parent who was born outside of the Netherlands and does not speak Dutch as their first language ${ }^{21}$. The influence of medical characteristics on IQ and selective attention at eight years of age was tested in two separate models. Diagnosis, type of ECMO, duration of mechanical ventilation and CLD were added into the multivariate linear regression analyses. The assumptions for multivariate linear regression analysis were checked with normal probability plots of the residuals and the Durbin-Watson test. Multicollinearity was evaluated using the criterion that variance inflation factors could not exceed $2.5^{23}$.

Analyses were performed with SPSS 22.0 (IBM, Chicago, IL, USA). For all analyses, a pvalue of $<.05$ was considered statistically significant. 


\section{Results}

Participants had a significantly higher birthweight than non-participants (mean birthweight (SD) $=3461$ (552) and 3294 (556) grams respectively; $p=.02$ ). No other clinical differences were found between participants and non-participants.

Developmental trajectory of intelligence

Intelligence fell within the normal range at two, five and eight years of age (Figure 2) ${ }^{13-15}$. Mauchly's test indicated that the assumption of sphericity had been violated $(p<.001)$, therefore Greenhouse-Geisser corrected tests are reported $(\varepsilon=.01)$. Intelligence was found to remain stable from two, to five, to eight years of age $(p=.15, \mathrm{n}=152)$. At eight years old, six children $(3 \%)$ had low IQ scores (<70), 39 children $(22 \%)$ had below average IQ scores $(\leq 85), 103$ children (58\%) had average IQ scores (85-115) and 30 children (17\%) had an above average IQ $(\geq 115)$.

\section{Outcome and type of education}

Intelligence

Sixty-five (37\%) of the ECMO survivors needed extra help at school at eight years versus 20\% of children in the general population ${ }^{24}$. Twelve children (7\%) in our cohort attended special education at eight years of age (Table $1 \mathrm{a}$ ), compared to $4.4 \%$ in the general population ${ }^{24}$. To get a better understanding of the relatively high proportions of children receiving extra help and attending special education, we analyzed its relationship with intelligence. Children attending special education had significantly lower intelligence from two years onwards, whereas those attending regular education, irrespective of their need for extra help, had comparable intelligence to the general population (Table 2).

\section{Selective attention}

The ECMO survivors who attended regular education without help performed significantly better on the selective attention task compared to those needing extra help or attending special education $(p=.02)$. Selective attention did not significantly differ between the ECMO survivors attending special education and those needing extra help $(p=.75)$ (Table 2).

\section{Diagnosis}

Intelligence did not differ at two and five years of age between MAS, CDH or other diagnoses. At eight years of age, $\mathrm{CDH}$ patients had a significantly lower IQ than those with other diagnoses, $(p=.04)$. Furthermore, significant differences were found on selective attention between the three diagnostic groups ( $p=.007$ ), with the CDH group scoring lowest on the selective attention task (Table 3).

Predictors of outcome at eight years

Low MEL increased the likelihood of having a lower IQ at eight years of age. Also, children with higher IQ scores at five years of age were more likely to have higher IQ at eight. Having a lower IQ score at five years of age increased the likelihood of a poorer score on the selective attention task at eight years of age (Table 4).

None of the medical characteristics were significantly related to outcome at eight years of age (Table 4). 


\section{Discussion}

This is the first longitudinal assessment of IQ in a large group of children treated with neonatal ECMO. We showed that intelligence falls within the average range at two, five and eight years of age and remains stable. This is in line with previous cross-sectional studies ${ }^{2,3,7,8,21}$. Strikingly, a large group of children attending regular education needed extra help in school, despite average intelligence. We found that these school difficulties were related to selective attention problems. As current follow-up protocols focus mainly on IQ, language and visuomotor integration domains that have been shown to remain intact following neonatal ECMO ${ }^{6,7,25-27}$ - those ECMO survivors at risk for school problems will not be identified. Our results underline the importance of standardized, evidence-based and problem-oriented neuropsychological follow-up following neonatal ECMO.

Despite the fact that the ECMO survivors included in this study did not have severe neurological morbidity, twice as many children in our cohort needed extra help at regular education compared to the general population ${ }^{24}$, as previously found by our group ${ }^{2}$. However, all had average intelligence, which did not differ from those who did not need help in school. Also, a relatively high number of ECMO survivors attended special education compared to the general population $^{24}$. These children had below average intelligence. Interestingly, both the ECMO survivors needing extra help and the ones attending special education performed significantly worse on the selective attention task compared to the ECMO survivors not needing help in school. Our findings therefore allow us to identify two groups of neonatal ECMO survivors who (without overt neurological deficits) are at risk of long-term school problems: those with lower IQ and related neuropsychological impairments and those with average IQ but with isolated neuropsychological deficits. For children attending special education, poor selective attention is more in accordance with - and may be partly explained by - their below average intelligence. The combination of which may lead to the need for special education. However, for those children needing extra help, isolated neuropsychological deficits may cause the need for educational support.

For identification of those at risk of school problems, especially of the ECMO survivors with average intelligence, problem-oriented neuropsychological assessment that goes beyond testing global cognitive functioning with the use of an IQ test ${ }^{10}$ is essential. Attention and (working) memory have been shown to be overlapping constructs that share much of the same pathways in the brain ${ }^{29}$. The attention problems observed in our cohort could therefore be accompanied by (working) memory deficits. Indeed, earlier studies have shown neuropsychological problems to lie mainly in the attention and memory domains in these children $^{2-4,28}$. It is therefore highly recommended that besides intelligence, both attention and memory, or executive functioning altogether, are focused on following neonatal ECMO. Using the current guidelines, neonatal ECMO survivors at risk for school problems will not be identified $^{6,7,25,27,30}$. We therefore propose a problem-oriented revision of follow-up protocols.

Since neuropsychological impairments in neonatal ECMO survivors have shown to emerge in childhood and to persist even into adolescence ${ }^{2-4}$, neuropsychological follow-up that extends beyond the age of five is crucial ${ }^{6}$. Neonatal follow-up of premature infants has shown early developmental assessments of high-risk infants to often be imprecise - especially for those with milder impairments that at a later age do affect their school performance ${ }^{31}$. It is likely that this is similar to neonatal ECMO follow-up. Moreover, neuropsychological testing beyond early childhood is important as these types of deficits at a later age may not only continue to hamper academic performance, but also affect the ability to participate in society and thus lead a 
fulfilling life ${ }^{4}$. However, as we have shown intelligence at five years of age to be highly predictive of IQ at eight, elaborate IQ assessment both at five and at eight may be redundant. To make most efficient use of time and resources, assessment of a full IQ can be considered at five years of age so that specified neuropsychological assessment can be conducted at eight years of age. At eight years, IQ can be screened with the use of a few subtests and, only if needed, a full IQ test can be administered. Such a problem-oriented approach will make risk stratification and early identification of those neonatal ECMO survivors at risk for school problems more feasible.

Finally, within follow-up of neonatal ECMO survivors, certain risk factors of impaired neurodevelopment should be taken into account. IQ at eight years old and selective attention were lower in $\mathrm{CDH}$ patients compared to children with other underlying diagnoses. These findings confirm earlier work demonstrating CDH patients to have generally worse outcomes ${ }^{2}$. Our proposal of problem-oriented and evidence-based neuropsychological follow-up therefore seems even more critical for this particular patient group. None of the other clinical characteristics studied were found to predict outcome at eight years of age, this is in line with previous findings $^{2,4}$. Low MEL significantly increased the likelihood of lower IQ at eight years of age. Although this result is not specific to neonatal ECMO survivors ${ }^{31,32}$, it is important to take into account during neonatal ECMO follow-up.

In this nationwide study we are the first to longitudinally assess intelligence in a large group of neonatal ECMO survivors. We have identified two specific groups of neonatal ECMO survivors who may be at risk for school problems: those with neuropsychological impairments despite having average intelligence and those with below average intelligence and neuropsychological impairments. As sources for educational support are available for all schools in the Netherlands, the number of children needing educational support or special education reported in this study are likely to be accurate. Furthermore, due to the high level of compliance, selection bias is highly unlikely. Nonetheless, our study has some limitations. First, a Dutch test measuring selective attention was used which limits cross-sectional comparisons. On the other hand, we were able to compare our data to Dutch normative data. Second, $11 \%$ of children $(n=31)$ did not complete the neuropsychological assessment at two and/or eight years of age due to cognitive or behavioral impairments $(n=14$, seen elsewhere with severe cognitive impairment (i.e. IQ <70); $n=11$, too tired or uncooperative at time of assessment; $n=6$, tested elsewhere but had average IQ scores), which may have resulted in a bias. However, the percentage of dropouts due to severe cognitive impairment was relatively low in comparison to the total number of participants, making significant bias unlikely. Third, treatment technologies, especially the use of centrifugal pumps, a smaller priming volume and new membranes with subsequently other adherence of commonly used drugs, are constantly changing and this may affect long-term outcomes. The current findings may thus not be generalizable to patients treated in recent years. Future studies should compare outcome between patients treated in different time periods to see what the effects of technology changes are in the long-term. Finally, at the time of data collection, our neuropsychological follow-up consisted only of intelligence and attention tests. Therefore, other cognitive functions that might be susceptible to impairment following neonatal ECMO, such as memory and executive functioning ${ }^{4}$, were not evaluated. Future studies should include these cognitive functions when assessing long-term neurodevelopment in neonatal ECMO survivors. 


\section{Conclusion}

Neonatal ECMO survivors have an overall average and stable IQ from two, to five, to eight years of age. Despite this, a large group is at risk for school problems. In the majority of ECMO survivors at risk, these school problems were related to isolated selective attention deficits. IQ alone is therefore not a reliable predictor of school performance or even eventual participation in society. As current neonatal ECMO follow-up protocols mainly focus on IQ and language and visuomotor integration, those children at risk will not be identified. Our findings emphasize the need for evidence-based, problem-oriented neuropsychological follow-up with a focus on attention and memory functioning following neonatal ECMO. Taken into account should be risk factors such as low MEL and/or a CDH diagnosis. As neuropsychological impairments have been shown to emerge in childhood and persist into adolescence, follow-up should extend beyond five years of age. 


\section{Acknowledgements}

We thank Joost van Rosmalen (PhD) from the department of Biostatistics (Erasmus MC, Rotterdam, the Netherlands) for statistical input and advice. 


\section{References}

1. Extracorporeal Life Support Organization. ECLS registry report, international summary January 2016. Ann Arbor, MI 2016.

2. Madderom MJ, Reuser JJ, Utens EM, van Rosmalen J, Raets M, Govaert P, et al. Neurodevelopmental, educational and behavioral outcome at 8 years after neonatal ECMO: a nationwide multicenter study. Intensive Care Med. 2013;39(9):1584-1593.

3. McNally H, Bennett CC, Elbourne D, Field DJ, Group UKCET. United Kingdom collaborative randomized trial of neonatal extracorporeal membrane oxygenation: followup to age 7 years. Pediatrics. 2006;117(5):e845-854.

4. Madderom MJ, Schiller RM, Gischler SJ, van Heijst AF, Tibboel D, Aarsen FK, et al. Growing Up After Critical Illness: Verbal, Visual-Spatial, and Working Memory Problems in Neonatal Extracorporeal Membrane Oxygenation Survivors. Crit Care Med. 2016. DOI: 10.1097/CCM.0000000000001626.

5. de Kleine MJ, den Ouden AL, Kollee LA, Nijhuis-van der Sanden MW, Sondaar M, van Kessel-Feddema BJ, et al. Development and evaluation of a follow up assessment of preterm infants at 5 years of age. Arch Dis Child. 2003;88(10):870-875.

6. The Extracorporeal Life Support Organization (ELSO). ELSO recommendations for follow-up for ECMO patients. https://www.elso.org/Portals/0/IGD/Archive/FileManager/2440a82ecdcusersshyerdocum entselsorecommendationsforneonatalpediatricecmopatientfollowup.pdf. Published 1994. Updated 01-97. Accessed 01-16.

7. Hanekamp MN, Mazer P, van der Cammen-van Zijp MH, van Kessel-Feddema BJ, Nijhuis-van der Sanden MW, Knuijt S, et al. Follow-up of newborns treated with extracorporeal membrane oxygenation: a nationwide evaluation at 5 years of age. Crit Care. 2006;10(5):R127.

8. Glass P, Wagner AE, Papero PH, Rajasingham SR, Civitello LA, Kjaer MS, et al. Neurodevelopmental status at age five years of neonates treated with extracorporeal membrane oxygenation. J Pediatr. 1995;127(3):447-457.

9. Bennett CC, Johnson A, Field DJ, Elbourne D, Group UKCET. UK collaborative randomised trial of neonatal extracorporeal membrane oxygenation: follow-up to age 4 years. Lancet. 2001;357(9262):1094-1096.

10. Aylward GP. Cognitive and neuropsychological outcomes: more than IQ scores. Ment Retard Dev Disabil Res Rev. 2002;8(4):234-240.

11. Stolar CJ, Snedecor SM, Bartlett RH. Extracorporeal membrane oxygenation and neonatal respiratory failure: experience from the extracorporeal life support organization. J Pediatr Surg. 1991;26(5):563-571.

12. Hofhuis W, Hanekamp MN, Ijsselstijn H, Nieuwhof EM, Hop WC, Tibboel D, et al. Prospective longitudinal evaluation of lung function during the first year of life after extracorporeal membrane oxygenation. Pediatr Crit Care Med. 2011;12(2):159-164.

13. Ruiter SAJ, Spelberg HCL, van der Meulen BF, Nakken H. The BSID-II-NL: Construction, standardisation, and instrumental utility. Netherlands Journal of Psychology. 2008;64(1):19-75.

14. Bleichrodt N, Drenth, P.J.D., Zaal, J.M., Resing, W.C.M. Intelligentiemeting bij Kinderen. : Lisse, Zwets en Zeitlinger; 1987. 
15. Kort W, Compaan, E.L. . WISC NL III. Handleiding. Amsterdam: NIP Dienstencentrum; 1999.

16. Madderom MJ, Toussaint L, van der Cammen-van Zijp MH, Gischler SJ, Wijnen RM, Tibboel D, et al. Congenital diaphragmatic hernia with(out) ECMO: impaired development at 8 years. Arch Dis Child Fetal Neonatal Ed. 2013;98(4):F316-322.

17. Vos P. Bourdon-Vos: handleiding.Lisse, Swets and Zeitlinger; 1992.

18. Centraal Bureau voor de Statistiek (Statistics Netherlands). Standaard Onderwijsindeling 2006 (The Dutch Standard Classification of Education). www.cbs.nl/nlNL/menu/methoden/classificaties/overzicht/soi/2003/default.html. Published 2006.

19. Jobe AH, Bancalari E. Bronchopulmonary dysplasia. Am J Respir Crit Care Med. 2001;163(7):1723-1729.

20. Kumar P, Shankaran S, Bedard MP, Delaney-Black V. Identifying at risk infants following neonatal extracorporeal membrane oxygenation. J Perinatol. 1999;19(5):367372.

21. Mazer P, Gischler SJ, MH VDC-VZ, Tibboel D, Bax NM, Ijsselstijn H, et al. Early developmental assessment of children with major non-cardiac congenital anomalies predicts development at the age of 5 years. Dev Med Child Neurol. 2010;52(12):11541159.

22. Stolar CJ, Crisafi MA, Driscoll YT. Neurocognitive outcome for neonates treated with extracorporeal membrane oxygenation: are infants with congenital diaphragmatic hernia different? J Pediatr Surg. 1995;30(2):366-371.

23. Allison P. Logistic regression using the SAS system: theory and application. New York: SAS Institute; 1999.

24. Koopman P LG, Karssen M, van der Meijden A, Petit R. Vervolgmeting 1 Kengetallen Passend Onderwijs. Amsterdam: Kohnstamm Instituut; 2015.

25. Johnson A, Field D, Elbourne D, Grant A, Davis D, Greenough A, et al. The collaborative UK ECMO trial: Follow-up to 1 year of age. Pediatrics. 1998;101(4):E1.

26. Khambekar K, Nichani S, Luyt DK, Peek G, Firmin RK, Field DJ, et al. Developmental outcome in newborn infants treated for acute respiratory failure with extracorporeal membrane oxygenation: present experience. Archives of Disease in Childhood - Fetal and Neonatal Edition. 2006;91(1):F21-F25.

27. van den Hondel D, Madderom MJ, Goedegebure A, Gischler SJ, Mazer P, Tibboel D, et al. Sensorineural hearing loss and language development following neonatal extracorporeal membrane oxygenation. Pediatr Crit Care Med. 2013;14(1):62-69.

28. Cooper JM, Gadian DG, Jentschke S, Goldman A, Munoz M, Pitts G, et al. Neonatal Hypoxia, Hippocampal Atrophy, and Memory Impairment: Evidence of a Causal Sequence. Cerebral Cortex. 2013.

29. Gazzaley A, Nobre AC. Top-down modulation: bridging selective attention and working memory. Trends Cogn Sci. 2012;16(2):129-135.

30. Khambekar K, Nichani S, Luyt DK, Peek G, Firmin RK, Field DJ, et al. Developmental outcome in newborn infants treated for acute respiratory failure with extracorporeal membrane oxygenation: present experience. Arch Dis Child Fetal Neonatal Ed. 2006;91(1):F21-25.

31. Moddemann D, Shea S. The developmental paediatrician and neonatal follow-up. Paediatr Child Health. 2006;11(5):295-299. 
32. Turkheimer E, Haley A, Waldron M, D'Onofrio B, Gottesman, II. Socioeconomic status modifies heritability of IQ in young children. Psychol Sci. 2003;14(6):623-628. 
Tables

Table 1. Patient characteristics

\begin{tabular}{|c|c|c|c|c|}
\hline & All $(\mathrm{n}=178)$ & MAS $(\mathrm{n}=97)$ & $\mathrm{CDH}(\mathrm{n}=36)$ & Other $(n=45)$ \\
\hline \multicolumn{5}{|l|}{ a) Demographic } \\
\hline \multicolumn{5}{|l|}{ Gender } \\
\hline Male & $96(54)$ & $46(47)$ & $24(67)$ & $26(58)$ \\
\hline Female & $82(46)$ & $51(53)$ & $12(33)$ & $19(42)$ \\
\hline \multicolumn{5}{|l|}{ Ethnicity } \\
\hline Dutch & $143(81)$ & 75 (78) & $31(86)$ & $37(82)$ \\
\hline Non-Dutch & 34 (19) & $21(22)$ & $5(14)$ & $8(18)$ \\
\hline Unknown & 1 & 1 & 0 & 0 \\
\hline \multicolumn{5}{|l|}{ MEL } \\
\hline Low & $45(27)$ & $21(23)$ & $11(34)$ & $13(30)$ \\
\hline Moderate & 65 (39) & $38(42)$ & $10(30)$ & $17(40)$ \\
\hline High & $56(34)$ & $31(35)$ & $12(36)$ & $13(30)$ \\
\hline Unknown & 12 & 7 & 3 & 2 \\
\hline \multicolumn{5}{|l|}{ Type of education at 8} \\
\hline Regular & $100(56)$ & $58(60)$ & $18(51)$ & $24(53)$ \\
\hline Regular with help & $65(37)$ & 36 (37) & 15 (43) & $14(31)$ \\
\hline Special education & $12(7)$ & $3(3)$ & $2(6)$ & $7(16)$ \\
\hline Unknown & 1 & 0 & 1 & 0 \\
\hline \multicolumn{5}{|l|}{ b) Clinical } \\
\hline Birthweight (grms) & 3461 (552) & $3512(551)$ & $3316(436)$ & 3465 (624) \\
\hline Gestational age (wks) & $40(2)$ & $41(2)$ & $39(1)$ & $39(2)$ \\
\hline \multicolumn{5}{|l|}{ Type of ECMO } \\
\hline VA & $155(87)$ & 77 (79) & $36(100)$ & $42(93)$ \\
\hline VV & $21(12)$ & $18(19)$ & $0(0)$ & $3(7)$ \\
\hline VV conversion to VA & $2(1)$ & $2(2)$ & $0(0)$ & $0(0)$ \\
\hline Unknown & 1 & 0 & 0 & 0 \\
\hline Age start ECMO (dys) & $1(0-23)$ & $1(0-10)$ & $1(0-11)$ & $2(0-23)$ \\
\hline Hours on ECMO & $140(24-369)$ & $135(24-288)$ & 177 (63-369) & $138(53-288)$ \\
\hline Mechanical vent. (dys) & $14(3-68)$ & $13(6-32)$ & $28(7-68)$ & $13(3-40)$ \\
\hline \multicolumn{5}{|l|}{ O2 post-ECMO } \\
\hline 1 day - 1 week & 87 (53) & $51(56)$ & $9(30)$ & $27(63)$ \\
\hline$>1$ week $-<1$ month & 64 (39) & 35 (39) & $14(47)$ & $15(35)$ \\
\hline$>1$ month & $13(8)$ & $5(5)$ & $7(23)$ & $1(2)$ \\
\hline Unknown & 14 & 6 & 6 & 2 \\
\hline \multicolumn{5}{|l|}{ CLD presence } \\
\hline Yes & $39(23)$ & $18(20)$ & $16(50)$ & $5(11)$ \\
\hline No & $129(77)$ & $74(80)$ & $16(50)$ & 39 (89) \\
\hline Unknown & 10 & 5 & 4 & 1 \\
\hline \multicolumn{5}{|l|}{ Abnormal CUS } \\
\hline Yes & $17(10)$ & $6(6)$ & $2(6)$ & $9(20)$ \\
\hline No & $159(90)$ & $91(94)$ & $33(94)$ & $35(80)$ \\
\hline Unknown & 2 & 0 & 1 & 1 \\
\hline
\end{tabular}


$\mathrm{N}(\%)$ is reported for all demographic variables. Non-Dutch refers to children with at least one non-native Dutch parent. The mean (SD) is reported for birthweight and gestational age. The median (range) is reported for age start ECMO in days, total amount of hours on ECMO and time on mechanical ventilation in days. $\mathrm{N}$ (\%) was reported for extra oxygen need post-ECMO, type of ECMO and CLD presence. Other diagnoses were sepsis $(n=10)$, persistent pulmonary hypertension of the newborn (PPHN; $n=30)$, pneumonia $(n=2)$, congenital cystic adenomatoid malformation of the lung $(n=1)$, pneumothorax $(n=1)$ and infant respiratory distress syndrome $(\mathrm{n}=1)$. Abbreviations: MAS, meconium aspiration syndrome; $\mathrm{CDH}$, congenital diaphragmatic hernia; MEL; maternal educational level; grms, grams; wks, weeks; dys, days; ECMO, extracorporeal membrane oxygenation; O2 post-ECMO, extra oxygen supply post-extubation; VA, venoarterial; VV, venovenous; CLD, chronic lung disease; CUS, cranial ultrasound.

Table 2. Outcome based on type of education attendance at eight years of age

\begin{tabular}{llll|l}
\hline & $\begin{array}{l}\text { MDI 2 yrs } \\
(\mathrm{n}=178)\end{array}$ & $\begin{array}{l}\text { IQ 5 yrs } \\
(\mathrm{n}=152)\end{array}$ & $\begin{array}{l}\text { IQ 8 yrs } \\
(\mathrm{n}=178)\end{array}$ & $\begin{array}{l}\text { Selective attention 8 yrs } \\
(\mathrm{n}=148)\end{array}$ \\
\hline Regular education & $105(16)$ & $106(14)$ & $104(16)$ & $-1.50(1.93)$ \\
Regular education with help & $100(19)$ & $95(17)$ & $95(15)$ & $-2.54(3.18)$ \\
Special education & $83(19)^{*}$ & $81(15)^{*}$ & $77(15)^{*}$ & $-2.91(2.21)$ \\
\hline
\end{tabular}

Mean (SD) of MDI/IQ and mean z-score (SD) of selective attention as measured by working speed are reported based type of education. Mean z-score (SD) = 0 (1). IQ population mean (SD) = 100 (15). *Significantly different IQ score than the general population at $p<.001$.

Abbreviations: MDI, mental developmental index; yrs, years.

Table 3. Neuropsychological outcome based on diagnosis

\begin{tabular}{llll|l}
\hline & MDI 2 yrs & IQ 5 yrs & IQ 8 yrs & Selective attention 8 yrs \\
\hline MAS & $104(18)$ & $101(14)$ & $100(15)$ & $-1.60(2.13)$ \\
$n$ & 97 & 81 & 97 & 86 \\
CDH & $98(18)$ & $98(21)$ & $93(20)^{*}$ & $-3.48(3.46)^{*}$ \\
$n$ & 36 & 32 & 36 & 30 \\
Other & $99(18)$ & $99(18)$ & $100(19)$ & $-1.39(1.88)$ \\
$n$ & 45 & 39 & 45 & 32 \\
\hline
\end{tabular}

Mean (SD) of MDI/IQ and mean z-Score (SD) of selective attention as measured by working speed are reported based on diagnosis. The IQ population mean $=100$ (15). Mean z-score (SD) $=0$ (1).

*Significantly different compared to other diagnostic groups at $p<.05$.

Other diagnoses include persistent pulmonary hypertension of the newborn, sepsis, cardinal respiratory insufficiency, persistent fetal circulation and respiratory syncytial infection.

Abbreviations: MDI, mental developmental index; MAS, meconium aspiration syndrome; $\mathrm{CDH}$, congenital diaphragmatic hernia; yrs, years. 
Table 4. Predictors of outcome at eight years.

\begin{tabular}{|c|c|c|}
\hline & IQ 8 yrs & Selective attention $8 \mathrm{yrs}$ \\
\hline \multicolumn{3}{|c|}{ Demographic predictors } \\
\hline Low MEL & $\begin{array}{l}B=-4.72, p=.03 \\
(C I-8.90--0.53)\end{array}$ & $\begin{array}{l}B=0.04, p=.94 \\
(C I-1.00-1.08)\end{array}$ \\
\hline Dutch ethnicity & $\begin{array}{l}B=-1.80, p=.45 \\
(\mathrm{CI}-6.51-2.92)\end{array}$ & $\begin{array}{l}B=-1.10, p=.09 \\
(\mathrm{CI}-2.27-0.15)\end{array}$ \\
\hline MDI at two & $\begin{array}{l}B=0.07, p=.22 \\
(\mathrm{CI}-0.04-0.18)\end{array}$ & $\begin{array}{l}B=-0.01, p=.54 \\
(\mathrm{CI}-0.04-0.02)\end{array}$ \\
\hline IQ at five & $\begin{array}{l}B=0.75, p<.001 \\
(C I 0.63-0.88)\end{array}$ & $\begin{array}{l}B=-0.07, p<.001 \\
(C I-0.10--0.04)\end{array}$ \\
\hline \multicolumn{3}{|l|}{ Medical predictors } \\
\hline $\mathrm{CDH}$ & $\begin{array}{l}B=-5.02, p=.25 \\
(C I-13.65-3.62)\end{array}$ & $\begin{array}{l}B=1.31, p=.07 \\
(\mathrm{CI}-0.08-2.70)\end{array}$ \\
\hline MAS & $\begin{array}{l}B=-0.43, p=.89 \\
(\mathrm{CI}-6.70-5.83)\end{array}$ & $\begin{array}{l}B=-0.01, p=.98 \\
(\mathrm{CI}-1.04-1.02)\end{array}$ \\
\hline VA & $\begin{array}{l}B=2.82, p=.49 \\
(C I-5.21-10.84)\end{array}$ & $\begin{array}{l}B=-0.10, p=.87 \\
(C I-1.27-1.08)\end{array}$ \\
\hline Mech. vent. (days) & $\begin{array}{l}B=-.19, p=.19 \\
(\mathrm{CI}-0.46-0.09)\end{array}$ & $\begin{array}{l}B=0.04, p=.06 \\
(\mathrm{CI}-0.00-0.09)\end{array}$ \\
\hline
\end{tabular}

Multivariate regression analyses to assess the influence of demographic and medical characteristics on outcome at eight years of age. Selective attention is measured by working-speed given in seconds; a higher score represents slower working-speed and vice versa. MEL (high MEL versus low and moderate MEL; low MEL versus high and moderate MEL) and diagnosis (CDH versus rest; MAS versus rest) are dummy variables (yes $=1$, no $=0$ ). Ethnicity is Dutch $(1)$ or non-Dutch $(0)$. A $\boldsymbol{P}$-value of $>.05$ was considered statistically significant.

Abbreviations: yrs, years; MEL, maternal educational level; MDI, mental developmental index; $\mathrm{CDH}$, congenital hernia diaphragmatic; MAS, meconium aspiration syndrome; VA ECMO, venoarterial extracorporeal membrane oxygenation; CLD, chronic lung disease. 


\section{Figures}

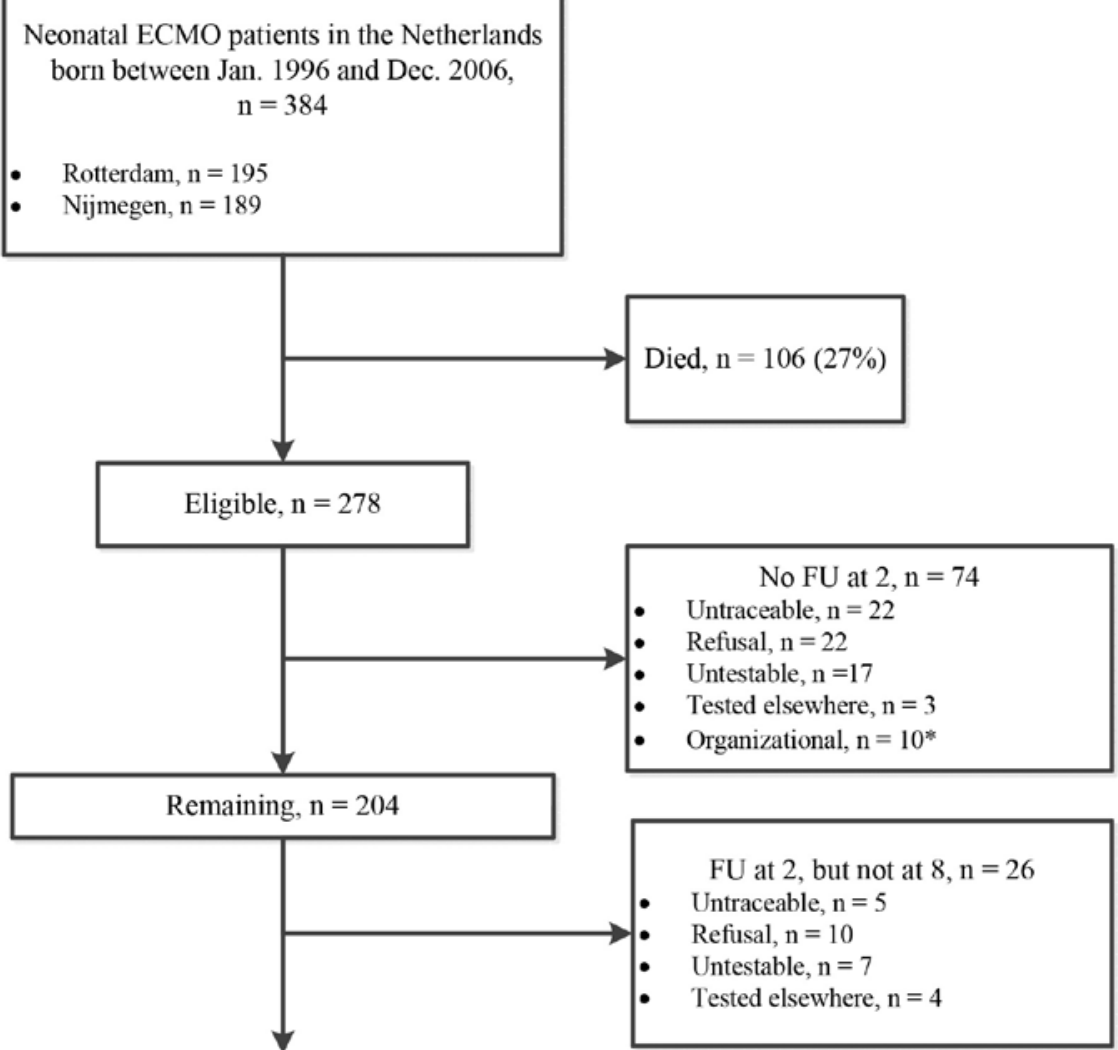

Follow-up at 2, 5 and 8 years of age, $\mathrm{n}=178$

- Rotterdam, $\mathrm{n}=96$

- Nijmegen, $\mathrm{n}=82$

\section{Figure 1.}

Inclusion flowchart of the neonatal ECMO survivors. 


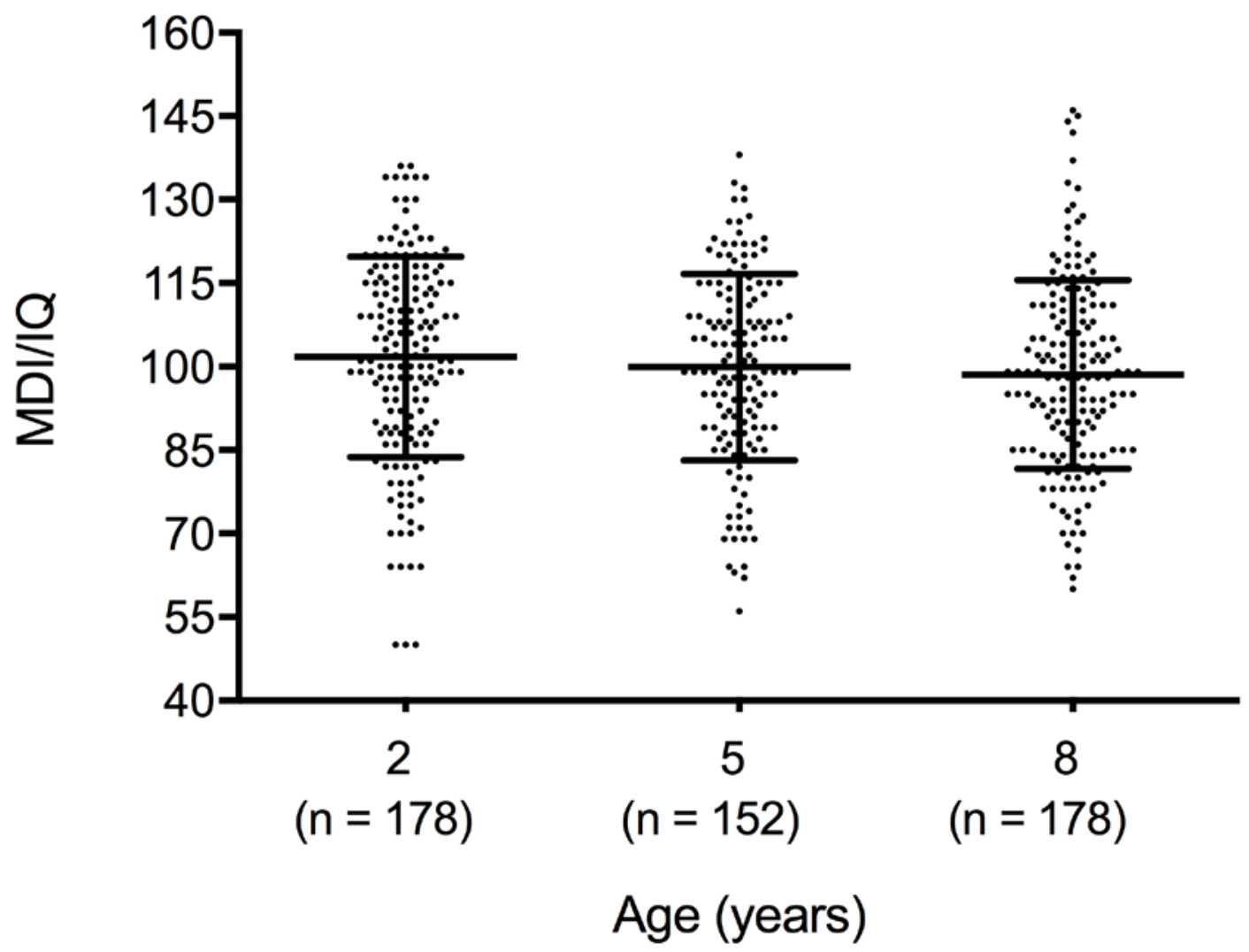

Figure 2.

Mean (SD) of MDI/IQ was reported at two, five and eight years of age. The population mean IQ $(\mathrm{SD})=100(15)$

Abbreviations: MDI, mental developmental index; IQ, intelligence quotient. 